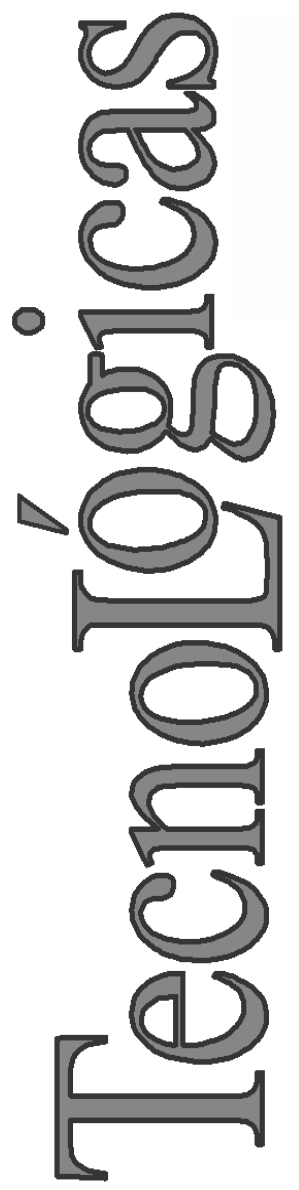

\title{
Análisis del Predictor de Smith Modificado
}

\section{Analysis of the Modified Smith Predictor}

\author{
Jorge A. Herrera-Cuartas ${ }^{1}$ \\ Julián Peláez-Restrepo ${ }^{2}$ \\ Nelson A. Correa-Rojas ${ }^{3}$
}

1 Grupo Automática, Electrónica y Ciencias Computacionales, Facultad de Ingenierías, Instituto

Tecnológico Metropolitano, Medellín-Colombia jorgeherrera@itm.edu.co

2 Grupo Automática, Electrónica y Ciencias Computacionales, Facultad de Ingenierías, Instituto

Tecnológico Metropolitano, Medellín-Colombia julianpelaez@itm.edu.co

3 Grupo Automática, Electrónica y Ciencias Computacionales, Facultad de Ingenierías, Instituto

Tecnológico Metropolitano, Medellín-Colombia nelsoncorrea@itm.edu.co 


\section{Resumen}

En el presente trabajo se presenta un análisis del predictor de Smith modificado, el cual es un esquema utilizado en el control de sistemas estables, inestables e integrativos. Se desarrolla y analiza la ecuación en lazo cerrado. Adicionalmente se realizan una serie de pruebas para verificar el desempeño de este esquema de control ante diferentes condiciones del modelo de la planta. Específicamente se realizan tres pruebas. En primer lugar, se verifica cómo se comporta el esquema ante una incertidumbre en el modelo del retardo, paso seguido se muestra el comportamiento del esquema ante variaciones de los parámetros de la planta.

\section{Palabras clave}

Predictor de Smith modificado; sistemas inestables; sistemas con retardo; compensación; predicción.

\section{Abstract}

In this paper an analysis about the modified Smith predictor, is presented. The modified Smith predictor is a scheme used to control stable, unstable and integrative systems. The closed loop equation is developed and analyzed. Additionally, various tests are made to verify the behavior of the control scheme. Specify, three tests are made. First, it is verify the behavior of the scheme to deal with an uncertainty in the delay model. Second, it is verify the behavior in the face of uncertainties in the parameter of the rational model.

\section{Keywords}

Modified Smith predictor; unstable systems; delay systems; compensation; prediction. 


\section{INTRODUCCIÓN}

El retardo es un fenómeno físico presente en todos los sistemas dinámicos, en algunas ocasiones su tamaño es despreciable y por lo tanto no afecta la dinámica del proceso. Existen diversos motivos por los cuales un sistema físico puede presentar retardo; éste se produce ya sea por las características intrínsecas del sistema o por factores externos que intervienen en él. Por características propias del sistema, podemos mencionar a modo de ejemplo, el transporte de masa o energía de un lugar a otro, tiempos de procesamiento y acumulación de pequeños retardos en sistemas conectados en serie, etc.

El efecto del retardo sobre el sistema está relacionado directamente con la relación entre la constante de tiempo del sistema y el tamaño del retardo. Diferentes enfoques se pueden utilizar para hacer frente al retardo. Por un lado, si el retardo es pequeño en comparación con la constante de tiempo del sistema, el controlador se puede diseñar haciendo una aproximación del retardo. Por otro lado, cuando el retardo es grande en comparación con la constante de tiempo del sistema, no se recomienda hacer una aproximación. En este caso el problema se puede enfocar usando un esquema de compensación del retardo como el predictor de Smith (PS).

El PS permite diseñar el controlador de una manera óptima aislando el retardo. Su principal inconveniente es su reducida robustez frente a incertidumbres en el modelo de la planta y su aplicación únicamente en sistemas estables. En (Majhi \& Atherton, 1999) presentan una extensión del PS para sistemas estables, inestables e integrativos. Recientemente, un enfoque basado en la identificación y control de sistemas con incertidumbre en el retardo ha sido propuesta para sistemas estables (Alcantara, Ibeas, Herrera, Vilanova, \& Pedret, 2009) (Herrera, Ibeas, Alcantara, \& de la Sen, 2011) e inestables (Herrera, Ibeas, Alcantara, \& Vilanova, 2010) (Herrera \& Ibeas, 2012). El enfoque presentado en (Alcantara, Ibeas, Herrera, Vilanova, \& Pedret, 2009) se basa en el PS y un esquema multimodelo. Este enfoque se extiende a sistemas estables, inestables e integrativos en (Herrera \& Ibeas, 2012). El enfoque tiene el mismo marco, pero en este caso, el esquema se basa en el predictor de Smith modificado (PSM) 
introducido en (Majhi \& Atherton, 1999). En estos trabajos por cuestión de espacio se presenta una breve introducción tanto del PS como del PSM pero en ningún caso se realiza un análisis profundo de su comportamiento y de sus propiedades en relación a incertidumbres en los parámetros del proceso.

Por lo anterior, este trabajo se centra en el análisis del PSM. Para ello se realiza la deducción analítica de la ecuación en lazo cerrado y se presenta las propiedades de estabilidad. Así mismo se realizan tres pruebas variando diferentes parámetros del esquema, con el fin de verificar el comportamiento del PSM. La primera prueba consiste en variar el retardo nominal del modelo. La segunda prueba consiste en variar los parámetros de la parte racional de la planta y finalmente se realiza un test donde se verifica que una variación en el retardo nominal puede mejorar la respuesta de un sistema con discrepancia en los parámetros de la parte racional.

El trabajo está organizado de la siguiente manera. En la Sección 2 se presenta una breve revisión del PS. En la Sección 3 se presenta el predictor de Smith modificado. Los ejemplos de simulación se presentan en la Sección 4. Finalmente, la Sección 5 concentra las principales conclusiones.

\section{PREDICTOR DE SMITH}

El punto de partida es el PS, mostrado en la Fig. 1, donde G(s) es el proceso y $e^{-h s}$ denota la función de transferencia asociada al retardo.

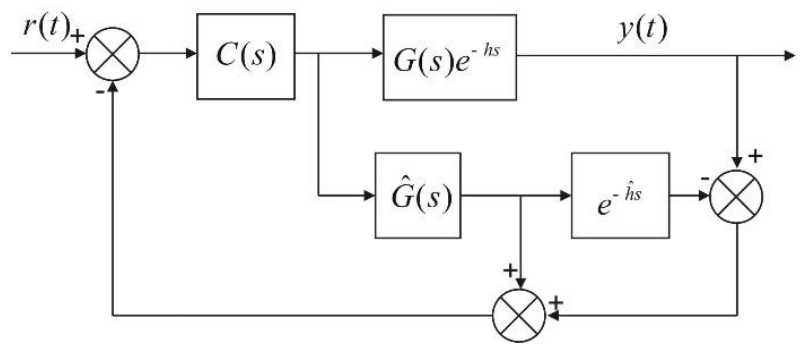

Fig. 1. Esquema básico del predictor de Smith. Fuente: Autores 
De acuerdo con el diagrama mostrado en la Fig. 1, la relación entrada-salida puede escribirse según:

$Y(s)=\frac{C(s) G(s) e^{-h s}}{1+C(s) \hat{G}(s)\left(1+e^{-h s}-e^{-\widehat{h} s}\right)} R(s)$

Donde $R(s)$ denota la transformada de Laplace de la señal de referencia $r(t)$ y C(s) denota la función de transferencia del controlador. A partir de (1) es sencillo obtener la ecuación característica del sistema, que determina su estabilidad, y que resulta ser:

$\operatorname{det}\left(1+C(s) \widehat{G}(s)\left(1+e^{-h s}-e^{-\widehat{h} s}\right)\right)=0$

Cuando $\hat{h}=h$, (2) se simplifica a:

$\operatorname{det}\left(1+C(s) G^{d f}(s)\right)=0$

de donde se observa que cuando el verdadero valor del retardo es utilizado en el esquema de control, la estabilidad del lazo cerrado viene proporcionada tan sólo por la conexión en realimentación de la componente racional de la planta (libre de retardo) y el compensador C(s). De esta forma, el compensador C(s) puede ser diseñado libremente sin tener en cuenta el retardo, lo cual simplifica el proceso de diseño del controlador.

\section{PREDICTOR DE SMITH MODIFICADO}

El PSM se muestra en la Fig. 2. Esta estructura se puede emplear para controlar procesos estables, inestables e integrativos, está compuesto de tres controladores los cuales son diseñados para diferentes objetivos.

El compensador $G_{c 1}$, en el lazo interno, se diseña para preestabilizar $\widehat{G}$ en el caso inestable. Los otros dos controladores: $G_{c}$ y $G_{c 2}$ se utilizan para el seguimiento a la referencia y el rechazo a perturbaciones, respectivamente, y se deben diseñar asegurando la 
estabilidad del sistema. Cuando $G_{c 1}=0$ y $G_{c 2}=0$, se obtiene la versión estándar del predictor de Smith mostrada en la Fig. 1.

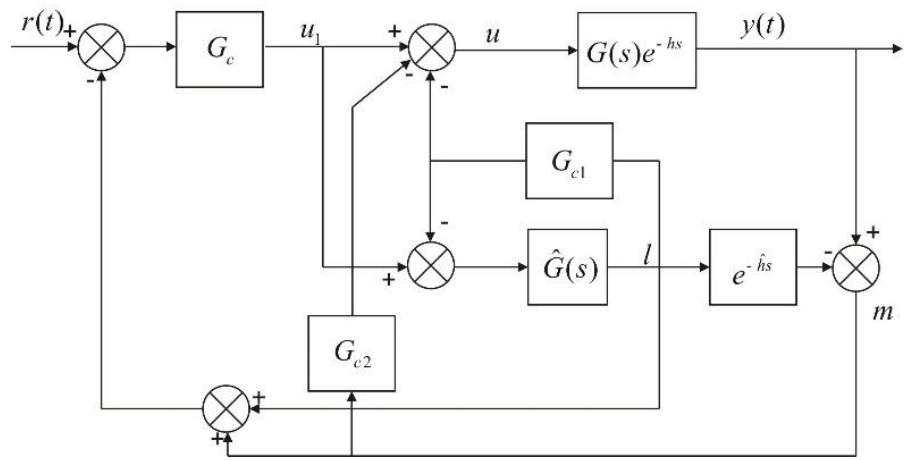

Fig. 2. Predictor de Smith modificado. Fuente: Autores

\subsection{Ecuación de Lazo Cerrado del Predictor de Smith Modificado}

Para obtener la respuesta en lazo cerrado se hace uso de las variables instrumentales $u_{1}, u, l$ y $m$, que se muestran en la Fig. 2.

La respuesta de lazo cerrado viene dada por:

$Y(s)=G e^{-h s}\left(u_{1}-G_{C 2} m-G_{C 1} l\right)$

Despejando $l$ y $m$ en términos de $u_{1}$, se tiene:

$l=\frac{\widehat{G} u_{1}}{1+G_{C 1} \hat{G}}, m=\frac{u_{1}\left(G e^{-h s}-\widehat{G} e^{-\widehat{h} s}\right)}{\left(1+G_{C 1} G^{d f}\right)\left(1+G_{C 2} G e^{-h s}\right)}$

Despejando $u_{1}$ en términos de la referencia, se tiene:

$u_{1}=G_{C}(R-m-l)$

Reemplazando (5) en (6), se obtiene:

$u_{1}=G_{C} R-\frac{G_{C} u_{1}\left(G e^{-h s}-\widehat{G} e^{-\widehat{h s}}\right)}{\left(1+G_{C 1} \hat{G}\right)\left(1+G_{C 2} G e^{-h s}\right)}-\frac{G_{C} \hat{G} u_{1}}{1+G_{C 1} \widehat{G}}$ 
Factorizando términos en común (7) se puede escribir como:

$u_{1}=\frac{G_{C} R\left(1+G_{C 1} \widehat{G}\right)\left(1+G_{C 2} G e^{-h s}\right)}{\left(1+\hat{G}\left[G_{c}+G_{c 1}\right]\right)\left(1+G_{C 2} \hat{G} e^{-h s}\right)+G_{c}\left(G e^{-h s}-\widehat{G} e^{-\widehat{h} s}\right)}$

Reemplazando (5) en (4) se obtiene:

$Y(s)=G e^{-h s}\left(u_{1}-\frac{G_{C 2} u_{1}\left(G e^{-h s}-\widehat{G} e^{-\widehat{h} s}\right)}{\left(1+G_{C 1} \widehat{G}\right)\left(1+G_{C 2} G e^{-h s}\right)}-\frac{G_{C 1} \hat{G} u_{1}}{1+G_{C 1} \widehat{G}}\right)$

Factorizando $u_{1}$ y reorganizando términos, se obtiene:

$Y(s)=\frac{G e^{-h s} u_{1}\left(1+G_{C 2} \hat{G} e^{-\widehat{h s}}\right)}{\left(1+G_{C 1} \hat{G}\right)\left(1+G_{C 2} G e^{-h s}\right)}$

Reemplazando (8) en (10) se obtiene la ecuación en lazo cerrado del PSM:

$Y(s)=\frac{G_{c} G e^{-h s}\left(1+G_{C 2} \widehat{G} e^{-\widehat{h} s}\right) R(s)}{\left(1+\hat{G}\left[G_{c}+G_{c 1}\right]\right)\left(1+G_{C 2} \widehat{G} e^{-h s}\right)+G_{c}\left(G e^{-h s}-\widehat{G} e^{-\widehat{h} s}\right)}$

En el caso que $\hat{h}=h$, entonces (11) se reduce a:

$Y(s)=\frac{G_{c} G e^{-h s} R(s)}{1+\widehat{G}\left[G_{c}+G_{c 1}\right]}$

Se puede ver como el sistema con retardo en la ecuación característica que aparece en (11), se convierte a un sistema con retardo externo en (12). Por lo tanto, este es una topología que desacopla el retardo de la estrategia de control, haciendo el sistema más fácil de controlar, dado que el controlador se puede diseñar sin tener en cuenta el retardo del sistema. 


\subsection{Propiedades de Estabilidad del Predictor de Smith Modificado}

Se demostrará que el PSM es robusto ante pequeñas incertidumbres en el retardo. Para ello se utiliza el siguiente Lema:

Lema 1: Considere que el PSM mostrado en la Fig. 1. con $G(s)=\widehat{G}(s)$ y $G_{C}(s), G_{C 1}(s)$ y $G_{C 2}(s)$ asintóticamente estable, dado que $G(s)$ es conocida y asintóticamente estable. Entonces existe un $\Delta \hat{h}$ tal que el sistema en lazo cerrado es asintóticamente estable cuando $\hat{G}(s)=G(s) e^{-(h+\Delta \hat{h}) s}$.

Demostración: La demostración está incluida en (Krstic, 2009) y (Niculescu, 2001).

Este resultado se puede interpretar diciendo que el PSM tiene un comportamiento robusto para pequeñas incertidumbres del retardo que no excedan $\Delta \hat{h}$.

\section{EJEMPLOS}

En esta sección se muestran simulaciones resultantes de aplicar el PSM. Se han considerado tres situaciones, en primer lugar se verifica la robustez del PSM ante incertidumbre en el retardo. En las tres primeras subsecciones se trata cada uno de estos tres casos asumiendo un conocimiento exacto de la dinámica de la planta libre de retardo. Para finalizar, se considera la situación en la que el modelo de la planta no describe de manera exacta el comportamiento de la planta real (discrepancias en la parte racional), observando el comportamiento robusto del esquema ante pequeñas incertidumbres en la planta.

El proceso para diseñar el controlador es realizado por (Majhi \& Atherton, 1999) para un proceso inestable de primer orden con retardo (FODUP, por sus siglas en inglés), el cual es descrito por (13). Siendo este modelo usual en muchos procesos industriales. 


$$
G(s)=\frac{K e^{-h s}}{\tau s-1}
$$

Donde $\tau, h$ y $K \in \mathbb{R}^{+}$.

\subsection{Variación del Retardo Nominal}

En esta sección se muestra el desempeño del PSM ante una incertidumbre en el retardo nominal. Para esta simulación se utilizaron los siguientes parámetros para la planta, el modelo de la planta y los controladores:

$G(s)=G^{d f}(s)=\frac{e^{-5 s}}{10 s-1}, G_{C}=\frac{0.5 s+1.7}{s}, G_{c 1}=13.4, G_{C 2} 2.4$

Donde $\hat{h} \in\{4.75,4.80,4.85,4.90,4.95,5.0,5.05,5.10,5.15,5.20,5.25\}$, $G_{C}$ es un controlador PI sintonizado con técnicas clásicas de sintonización de PID's. $G_{C 1}$ y $G_{C 2}$ se diseñaron teniendo en cuenta las recomendaciones dadas por (Majhi \& Atherton, 1999).

En la Fig. 3 se muestra la señal de salida para los diferentes valores del retardo. La línea negra muestra el valor de la salida para un retardo $\hat{h}=h$, es decir para el caso donde se conoce con exactitud el retardo de la planta. Se puede ver que se presenta un buen seguimiento de la referencia. Las simulaciones en rojo y en azul presentan el desempeño para una variación del retardo, se puede ver que a medida que la incertidumbre aumenta en el modelo del retardo la respuesta se va deteriorando considerablemente.

En la Fig. 4. Se muestra la señal de control para los diferentes casos, se puede ver claramente como aumenta la oscilación de la señal de control a medida que aumenta la discrepancia entre el verdadero retardo y el retardo nominal.

En la Tabla 1 se presenta el valor que toma la respuesta para la integral de error cuadrática (ISE, por sus siglas en ingles). En este punto se puede ver que a medida que la discrepancia entre el verdadero retardo y el nominal aumenta, el ISE aumenta exponencialmente. 


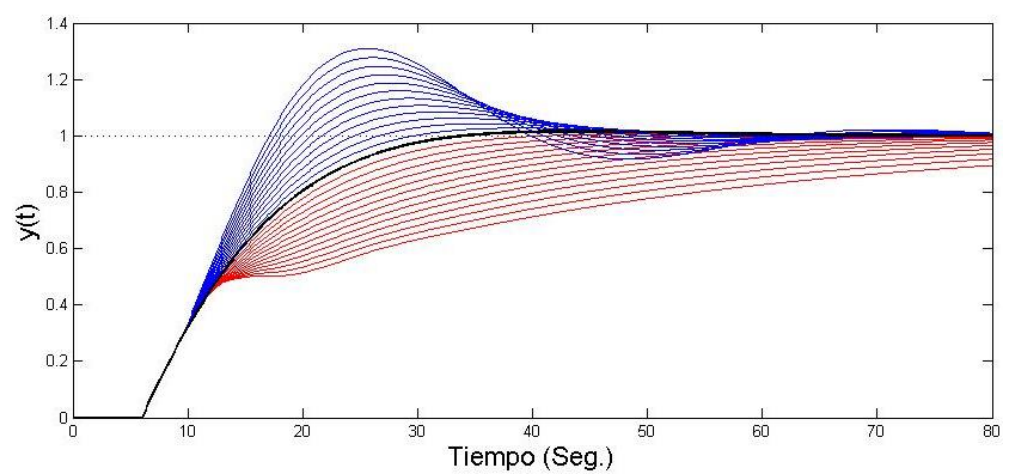

Fig. 3. Señal de salida variando el retardo nominal de 0 a $\pm 40 \%$. Fuente: Autores

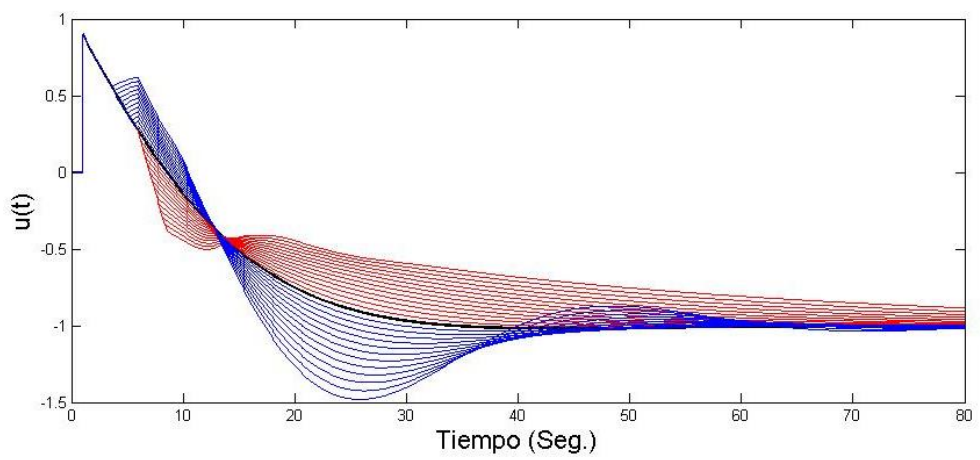

Fig. 4. Señal de control variando el retardo nominal de 0 a $\pm 5 \%$. Fuente: Autores

Tabla 1. Variación del ISE en relación con el retardo. Fuente: Autores

\begin{tabular}{rcccccccc}
\hline$h-\hat{h}$ & 0.05 & 0.10 & 0.15 & 0.20 & -0.05 & -0.10 & -0.15 & -0.20 \\
\hline ISE & 0.058 & 0.215 & 0.448 & 0.742 & 0.069 & 0.303 & 0.753 & 1.481 \\
\hline
\end{tabular}

\subsection{Robustez ante Variación en el Modelo de la Planta}

En esta sección se muestra el desempeño del PSM ante variaciones en los parámetros de la parte racional. Para esta prueba se utilizaron los parámetros descritos en (15), a diferencia que el modelo nominal toma los siguientes valores: 
$G^{d f}(s)=\frac{\widehat{K} e^{-5 s}}{\hat{\tau} s-1}$

Donde $\hat{k} \in\{0.80,0.81, \ldots, 1.20\}$ y $\hat{\imath} \in\{9.80,9.81, \ldots, 10.20\}$. En la Fig. 5 se muestra la señal de salida para los diferentes valores de los parámetros de la parte racional. La línea negra muestra el valor de la salida para un sistema perfectamente modelado. Las simulaciones en rojo y en azul presentan el desempeño ante variaciones de los parámetros de la planta. Al igual que en el caso anterior, se puede ver que a medida que aumenta la discrepancia entre los parámetros del sistema real y el modelo, la respuesta del sistema se deteriora llegando incluso a la inestabilidad.

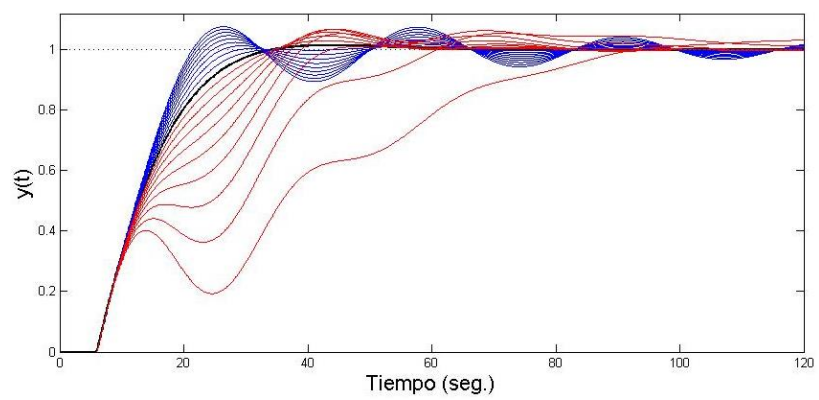

Fig. 5. Señal de salida variando los parámetros de la planta. Fuente: Autores

En la Fig. 6 se muestra la señal de control para el segundo caso, se ve como esta señal oscila a medida que aumenta la incertidumbre en el modelo nominal de la planta.

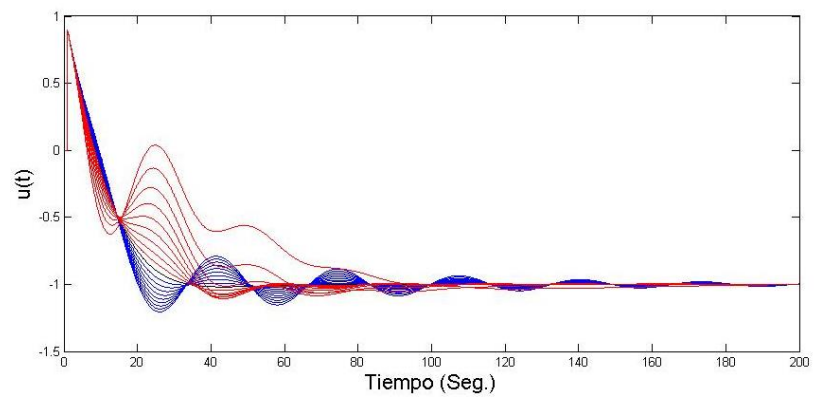

Fig. 6. Señal de control variando los parámetros de la planta. Fuente: Autores 
En la Tabla 2 se muestra el ISE para las diferente simulaciones, se puede observar claramente que este índice aumenta directamente con la discrepancia entre los modelos de la planta.

Tabla 2. Variación del ISE en relación con la variación de los parámetros.

Fuente: Autores.

\begin{tabular}{ccccccccc}
\hline$k$ & 0.98 & 0.97 & 0.96 & 0.95 & 1.01 & 1.02 & 1.03 & 1.04 \\
\hline$\tau$ & 10.02 & 10.03 & 10.04 & 10.05 & 9.99 & 9.98 & 9.97 & 9.96 \\
ISE & 0.246 & 0.557 & 0.998 & 1.575 & 0.0614 & 0.246 & 0.556 & 0.994 \\
\hline
\end{tabular}

\subsection{Compensación de la incertidumbre en la parte racional con un desfase en el retardo}

Al realizar las pruebas se ha visto que el error presentado en la salida del sistema debido a la incertidumbre en los parámetros de la parte racional se puede reducir de cierto modo con un desfase en el valor del retardo nominal.

La confirmación de esta hipótesis se muestra en la Fig. 7. La línea negra representa la salida del sistema perfectamente modelado. La línea roja representa la señal de salida con una discrepancia en los parámetros de la parte racional de la planta y con el retardo nominal perfectamente modelado. La línea azul presenta la salida con un desfase en el retardo de 0.2 segundos. Se puede ver que la respuesta presenta un tiempo de establecimiento menor a pesar que presenta un sobre pico mayor.

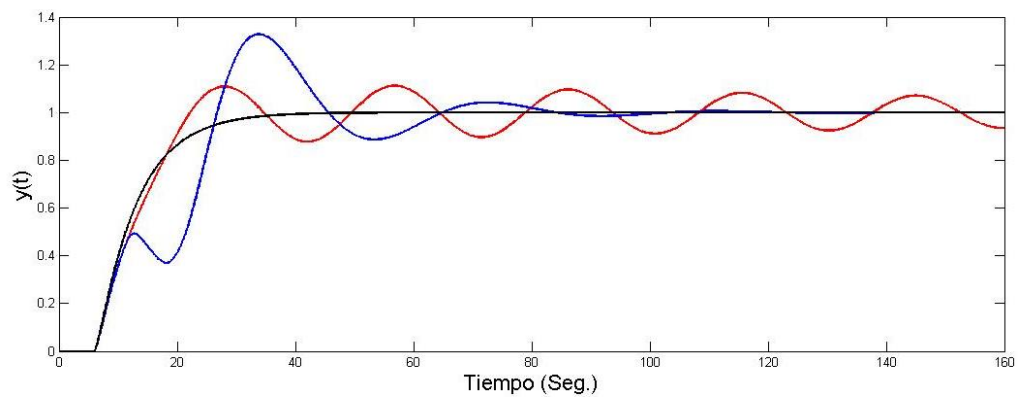

Fig. 7. Señal de salida con desfase en el retardo. Fuente: Autores 
Se realizaron diferentes simulaciones para diferentes valores del desfase del retardo, esto con el fin de caracterizar el esquema. Los resultados de estas simulaciones se muestran en la Fig. 8. En esta figura se muestra el ISE para diferentes valores del desfase del retardo. Se puede ver claramente como la curva tiene un mínimo global en 5.2 Seg.

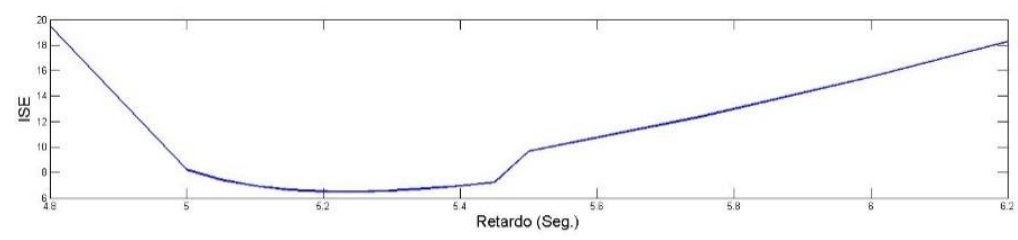

Fig. 8. ISE para diferentes valores del retardo. Fuente: Autores

\section{CONCLUSIONES}

En el presente artículo se ha presentado un análisis del predictor de Smith modificado. Se ha deducido analíticamente la ecuación en lazo cerrado y se ha enmarcado en una propiedad de estabilidad. Las pruebas que se presentan permiten verificar el comportamiento del PSM ante variaciones en el modelo de la planta. Y se ha corroborado que una incertidumbre en los parámetros de la planta, se pueden mitigar con un desfase en el retardo del modelo.

\section{AGRADECIMIENTOS}

Este trabajo fue parcialmente soportado por el grupo de investigación en Automática y electrónica del Instituto Tecnológico Metropolitano (ITM) bajo el proyecto "Maximización de extracción de energía en aerogeneradores para cogeneración urbana en el Valle de Aburrá", código P10-233. 


\section{REFERENCIAS}

Alcantara, S., Ibeas, A., Herrera, J., Vilanova, R., \& Pedret, C. (2009). Multi-model Smith predictor based control of multivariable systems with uncertain bounded external delays. IEEE Latin America Transactions, 7(1), 42-53.

Herrera, J., \& Ibeas, A. (2012). On-line delay estimation for stable, unstable and integrating systems under step response. ISA Transactions, $51,351-361$.

Herrera, J., Ibeas, A., Alcantara, S., \& de la Sen, M. (2011). Multimodelbased techniques for the identification and adaptive control of delayed multi-input multi-output systems. IET Control Theory \& Applications, 5(1), 188-202.

Herrera, J., Ibeas, A., Alcantara, S., \& Vilanova, R. (2010). Identification and adaptive control of delayed unstable systems. In: Proceedings of the 2010 international symposium on intelligent control.

Krstic, M. (2009). Delay Compensation for Non linear, Adaptive, and PDE Systems. Birkhauser.

Majhi, S., \& Atherton, D. (1999). Modified Smith predictor and controller for processes with time delay. IET Control Theory \& Applications, $146,359-66$.

Niculescu, S. I. (2001). Delay Effects on Stability. A Robust Control Approach. Springer-Verlag. 